Archives

14-15 | 1995

Les banques de données au $\mathrm{CRH}$

\title{
Le catasto florentin et son environnement
}

\author{
Christiane Klapisch-Zuber
}

\section{(2) OpenEdition}

Journals

\section{Édition électronique}

URL : http://journals.openedition.org/ccrh/2659

DOI : $10.4000 /$ ccrh.2659

ISSN : $1760-7906$

Éditeur

Centre de recherches historiques - EHESS

Édition imprimée

Date de publication : 14 octobre 1995

ISSN : 0990-9141

Référence électronique

Christiane Klapisch-Zuber, "Le catasto florentin et son environnement », Les Cahiers du Centre de Recherches Historiques [En ligne], 14-15 | 1995, mis en ligne le 27 février 2009, consulté le 20 avril 2019. URL : http://journals.openedition.org/ccrh/2659; DOI : 10.4000/ccrh.2659

Ce document a été généré automatiquement le 20 avril 2019

Article L.111-1 du Code de la propriété intellectuelle. 


\title{
Le catasto florentin et son environnement
}

\author{
Christiane Klapisch-Zuber
}

1 Entre 1966 et 1976, l'historien américain David Herlihy (disparu en 1991) et moi-même avons travaillé à engranger dans l'ordinateur, puis à exploiter un ensemble de données concernant la Toscane de 1427. Imposant par sa masse archivistique et la richesse de ses informations, il consistait en la description de la population et des biens des contribuables alors soumis à la domination de la Commune de Florence. Chacun d'entre eux devait décrire son patrimoine foncier et en estimer la valeur ou la rente. Mais, à la différence de la presque totalité des descriptions fiscales médiévales et modernes, il lui fallait remettre une déclaration même s'il ne possédait rien ; il devait aussi énumérer ses sources de revenus autres que foncières; enfin, il lui incombait de présenter une liste de tous les membres de son foyer en donnant de chacun le sexe et l'âge ainsi que le lien de parenté qui l'unissait à lui-même, le chef de famille. Arrivés au terme de ce long dépouillement, nous devions compter autour de 264000 personnes des deux sexes, réparties entre environ 60000 feux. A raison de dix-huit informations socio-économiques par foyer et de cinq informations par individu, nous arrivions à un total de plus d'un million pour les premières, et de 1300000 pour les secondes, évidemment couplées sous le nom d'un même titulaire.

2 L'analyse de cet ensemble impressionnant fut livrée dans un ouvrage écrit de concert et paru en $1978^{1}$. Le matériau, jusque là, avait surtout été considéré, dans des recherches ponctuelles, comme l'annuaire d'une ville illustrée par ses artistes, ses hommes d'État ou ses penseurs, ses compagnies de banque ou de commerce, quelques familles distinguées. Notre exploitation prétendait au contraire prendre les mesures d'une population dans toute son épaisseur et sa diversité régionale et sociale. L'ouvrage ne répondait certes pas à toutes les interrogations que pouvaient soulever sa matière et son propos; notre enquête dans les profondeurs de la société et de la famille toscanes n'épuisait pas davantage le riche matériau des archives fiscales florentines. Il suffit de rappeler que nous n'avions retenu qu'une partie limitée des informations de ce dénombrement-là et 
que la rédaction d'autres relevés du même type fut répétée, au moins à Florence, à un rythme d'abord serré, puis plus lâche, jusque dans les dernières décennies du $\mathrm{Xv}^{\mathrm{e}}$ siècle.

Et que dire des autres gisements d'archives toscans pouvant éclairer un aspect des structures, de la vie et de l'évolution d'une société, aspects que les historiens de la fin du Moyen Age n'entrevoient généralement que par des informations limitées ou confuses? Naissances et baptêmes, mariages et investissements dotaux, décès et sépultures, s'éclairent ainsi par les descriptions de banquets, trousseaux, cadeaux qui leur sont liés. Que l'initiative en vienne de la société civile ou des institutions publiques, on multiplie les inventaires ou descriptions de biens. Meubles, livres, cuirasses, tasses d'argent, que saisje? tout est récapitulé à des fins patrimoniales ou fiscales, même les biens les plus fragiles, les habits par exemple, qui font l'objet de minutieuses investigations, lorsqu'ils tombent sous le coup d'une loi somptuaire particulièrement vigilante ${ }^{2}$. Les listes publiques du personnel engagé dans la vie politique ou administrative de la Commune (des plus prestigieux politiciens aux hommes d'armes, chevaliers, trompettes ou horlogers de la Commune, jusqu'aux serviteurs retraités de l'État) trouvent leur contrepoint dans les indices d'échanges sociaux beaucoup plus ténus, irréguliers, malaisés à lire, que nous les entrevoyions dans les archives de la répression (listes d'exécutions capitales, de grâces ou condamnations) ou dans les traces qu'en conserve la mémoire privée (vendettas, services rendus et à rendre, que consignent les livres de famille à l'intention des descendants)... Il n'est guère de domaine de l'activité humaine qui n'ait été soumis à classement, enregistrement et évaluation par ces inlassables comptables que furent les Florentins. L'imagination descriptive alliée à la fureur bureaucratique a créé un océan de données largement indompté.

Mais il ne s'agit évidemment pas de tout saisir indistinctement, de céder à une boulimie documentaire accumulant des données inutilement. Je n'ai évoqué ces richesses que pour susciter l'appétit et montrer l'environnement des fonds les plus cohérents et massifs. Alors, que faire? Il y a certainement encore de beaux jours pour les chercheurs de tout poil, ne serait-ce qu'à travailler sur les corpus informatisés existants. Regroupés en une banque générale de données qui, un jour que j'espère proche, devrait se mettre en place, ces derniers peuvent encore ouvrir sur des perspectives excitantes de recherche. Les chercheurs qui, à l'ancienne, continueront de voir dans une banque de données l'index nominatif d'un dictionnaire dont une seule notice les intéressera; ceux qui, protégés par le matelas des données - mais l'espèce semble en voie de disparition - encaisseront les coups portés à la tradition d'une histoire quantifiée et massive ; ceux, plus aventureux, qui testeront des hypothèses pointues sur des ensembles numériquement larges et statistiquement fiables.

5 L'édifice pourrait se construire avec des matériaux variés. Quant au catasto même de 1427, une utilisation plus subtile de ses données individuelles exigerait que leur présentation en devienne complètement nominative, travail que les difficultés à traiter un matériel aussi énorme semblaient rendre inutile en 1966 ; l'entreprise avait été écartée, mais il serait possible de la reprendre à peu de frais et de compléter le fichier en y introduisant les données onomastiques complètes. D'autres catasti, postérieurs à celui de 1427, sont ou devraient être préparés de la même manière : celui de 1480 a dès maintenant été mis en machine et étudié par A. Molho et C. Corsini ${ }^{3}$; celui de 1457 est en cours de saisie. Ainsi, le $\mathrm{XV}^{\mathrm{e}}$ siècle serait balisé à trois moments importants de son histoire démographique et fiscale. La saisie des données anthroponymiques complètes ouvrirait la voie à des études longitudinales aujourd'hui à peu près impossibles. Prolongés en amont par les estimi de la 
seconde moitié $\mathrm{du} \mathrm{XIV}^{\mathrm{e}}$ siècle étudiés par A. Stella ${ }^{4}$, les catasti du Xve siècle constituent le mole auquel peuvent s'arrimer des recherches plus limitées, telles les reconstitutions de familles opérées à partir des livres de famille privés, qui à ce jour en ont corroboré ou affiné les résultats.

D'autres fonds, aux visées différentes, se sont déjà prêtés à l'utilisation informatique, ou pourraient bientôt le faire. Anthony Molho et Julius Kirshner ont mené à bien la saisie du Monte delle doti $i^{5}$. Ce fonds d'État destiné à encourager la nuptialité par son aide à la constitution des dots, entraîna l'inscription de dizaines de milliers de filles depuis un âge tendre. Il éclaire non seulement l'histoire financière, fiscale et politique de la république florentine aux $\mathrm{XV}^{\mathrm{e}}-\mathrm{XVI}^{\mathrm{e}}$ siècles, mais le destin de ces femmes jusqu'à leur mariage ou leur entrée en religion, parfois jusqu'à leur veuvage. Les registres des baptêmes citadins, en série continue après 1450 et jusqu'à la fin du XVIII ${ }^{\mathrm{e}}$ siècle, les livres de sépultures tenus depuis la fin $\mathrm{du}_{\mathrm{XIV}}{ }^{\mathrm{e}}$ siècle quoique de façon plus lacunaire et irrégulière, se prêteraient aussi à une entreprise systématique de collecte et mise en forme pour l'ordinateur. L'offre, du reste, ne s'arrête pas aux archives concernant l'histoire de la population ou de la fiscalité. David Herlihy avait avant sa mort entré en ordinateur les données concernant toute la classe politique florentine entre $\mathrm{XIV}^{\mathrm{e}}$ et $\mathrm{XVI}^{\mathrm{e}}$ siècle; le corpus repose sur la constitution des «bourses» contenant les noms des citoyens habilités aux offices municipaux, les tirages au sort et les élections aux postes d'administration ou de gouvernement. Ces archives dites des Tratte permettent de reconstituer toute la gamme des "carrières" politiques dans la république florentine et d'envisager l'étude prosopographique de toutes les catégories de citoyens, de rang modeste ou éminent, prenant part à l'administration et au gouvernement de l'État ${ }^{6}$. En aval, ce travail est prolongé par celui de R. Burr Litchfield (Brown University), qui a exploité les données sur les carrières bureaucratiques de la période grand-ducale?

Rêvons. L'accès sur réseau à ces fonds documentaires informatisés - et j'en passe - et la menée ou la coordination des enquêtes permises par les techniques modernes de communication laissent entrevoir le moment où la collaboration internationale se passera de son pesant appareil de savantes correspondances, de déplacements onéreux en temps, fatigue et argent, d'acrobaties financières entre bureaucraties de traditions différentes. Faut-il les regretter? D'autres types de convivialité scientifique sont à imaginer. Et ce n'est pas la perspective la moins excitante dans cette affaire.

\section{NOTES}

1. D. Herlihy et C. Klapigch-Zuber, Les Toscans et leurs familles. Une étude du catasto florentin de 1427, Paris, Editions de l'EHESS et Presses de la FNSP, 1978.

2. Ainsi le registre de 1344 actuellement étudié par Laurence Gérard-Marchand.

3. A. Molho, dans l'ouvrage cité à la note suivante ; C. Corsini, « La demografia fiorentina nell'età di Lorenzo il Magnifico ", Actes du congrès dédié à Lorenzo il Maqnifico, à paraître.

4. A. Stella, La révolte des Ciompi. Les hommes, les lieux, le travail, Paris, Éditions de l'EHESS, 1993. 
5. Outre leurs nombreux articles sur des points particuliers, cf. maintenant, sur le problème de l'endogamie sociale, l'étude de A. Molho, Marriage Alliance in Late Medieval Florence, Harvard University Press, 1994.

6. Une première analyse de ces fonds a été donnée par D. Herlihy, "The Rulers of Florence, 1282-1530» [1991], réed. in A. Molho, Women, Family and society in Medieval Europe. Historical Essays, 1978-1991, Providence et Oxford, Berghahn Books, 1995, p. 353-380.

7. R. B. Litchfield, Emergence of a Bureaucracy. The Florentine Patricians, 1530-1790. Princeton, 1986. 